\title{
Accretion disk and stream origin of the dip phenomenon in Hercules X-1
}

\author{
D. A. Leahy ${ }^{1}$ and C. D. Igna ${ }^{2}$ \\ ${ }^{1}$ Department of Physics and Astronomy, University of Calgary, \\ Calgary, Alberta, T2N 1N4, Canada \\ email: leahy@ucalgary.ca \\ ${ }^{2}$ Dept. of Astronomy, University, \\ add USA \\ email: zz@asxxxe
}

\begin{abstract}
The Rossi X-ray Timing Explorer has observed Hercules X-1 over a period of about 13 years, allowing detailed measurement of the properties of the dip phenomenon. We construct a model for the dips based on the impact between the accretion stream and the disk, including the geometry and dynamics of the stream and disk. We also consider the case where the stream penetrates the outer parts of the disk, where its density is low, and proceeds to a second impact at smaller radius in the disk. The results from the model are discussed and compared to the observed properties of the dips.
\end{abstract}

Keywords. Accretion, accretion disks, X-rays: binaries, stars: neutron

\section{Introduction}

The Hercules X-1 X-ray binary system consists of a neutron star (Her X-1) and its A7 companion (HZ Her). The binary has a 1.7-day orbit and exhibits eclipses of the neutron star. There is a longer term 35-day cycle in its X-ray flux which was attributed to a twisted tilted accretion disk and which was modelled by Petterson(1975). The neutron star accretes via the Roche-lobe overflow mechanism. HZ Her has expanded beyond it main sequence size and is in the early part of its hydrogen shell-burning phase, as discussed by Igna (2011).

\section{35-day cycle and accretion disk}

The 35-day X-ray cycle has been the object of many studies. The start times (called turn-on's) of the cycle are not strictly periodic, so comparing data on Her X-1 taken at widely spaced times (>35 days) has been difficult. The Rossi X-ray Timing Explorer (RXTE) All-Sky Monitor (ASM) data for Her X-1 was put together by Scott \& Leahy(1999) using 35-day turn-ons from the Compton Gamma-Ray Observatory (CGRO) Burst And Transient Source Experiment (BATSE), which monitored pulsations from the brighter X-ray pulsars nearly continuously, and thus could detect when the 35-day cycle main high states turned on and off. The resulting RXTE/ASM 2-12 keV average 35 day light curve of Her X-1 produced from a few years of data was the highest quality 35-day light curve for Her X-1 and showed many features in more detail. The 35-day cycle has a sequence of 4 states: main-high state; low-state; short-high state; low-state. This 35-day cycle is caused by periodic blockage of the X-rays from the neutron star by the accretion disk.

The twisted-tilted disk model put forward by Petterson(1975), had its geometry determined in detail by Leahy(2002) from models for the average RXTE/ASM 35-day light 
curve. This same disk also gives the changes of the X-ray pulse profile over 35-day cycle as shown by Scott et al.(2000). The 35-day light curve is sensitive to the disk shape, and the changes in pulse profile are sensitive to the geometry of the accretion column on the neutron star. This allowed inferring that the accretion column emitted a pencil beam normal to the neutron star surface and a fan beam horizontally to the surface. Detailed modeling of the pulse profile with an accretion column model was done by Leahy(2004a) including gravitational light bending and allowed the column geometry to be determined. Further modeling with variable mass and radius parameters by Leahy(2004b) allowed a tight mass-radius constraint for the neutron star.

The turn-on times for 147 of 35-day cycles during the RXTE lifetime were determined from the RXTE/ASM data by Leahy \& Igna(2010) using a cross-correlation analysis. Turn-on times and uncertainties and 35-day cycle lengths were measured (Table 1 in that paper). It was found that there was no evidence for turn-on's at specific orbital phases, such as 0.2 and 0.7 often mentioned in the literature. Rather the turn-on's are distributed uniformly in orbital phase.

In addition to the 35-day cycle in observed X-ray flux, the disk also causes a 35-day cycle in X-ray illumination and heating of the companion star. The heating was long ago observed in optical wavelengths, by Gerend \& Boynton(1976). Recently, the 35-day heating cycle was observed at Extreme Ultraviolet wavelengths by Leahy(2003).

RXTE observed Her X-1 numerous times with the large area- high sensitivity Proportional Counter Array(PCA). A summary of the observations is given by Leahy \& Igna(2011). For these data, the standard 2 data (with 16 second time bins) consists of 98906 time bins on 163 different MJDs, for a total net exposure time of 1.58 million s. Of the 147 cycles covered by RXTE/ASM observations, 37 cycles have PCA data in them.

The 35-day cycle and orbital cycle light curves were the focus of that study. The turnon times and cycle lengths from Leahy \& Igna(2010) were used to determine 35-day phase for all of the PCA observations. Figure 2 here shows a new composite figure of all $\mathrm{RXTE} / \mathrm{PCA}$ count rates in the $2-4 \mathrm{keV}$ band vs. 35-day phase. The main high state is from phase 0 to 0.31 and the short high state from phase 0.57 to 0.74 . The low states fill in the other 35-day phases. At any 35-day phase the count rate can go to zero- this is caused by the dip phenomenon- cold matter absorption lasting anywhere from a few minutes

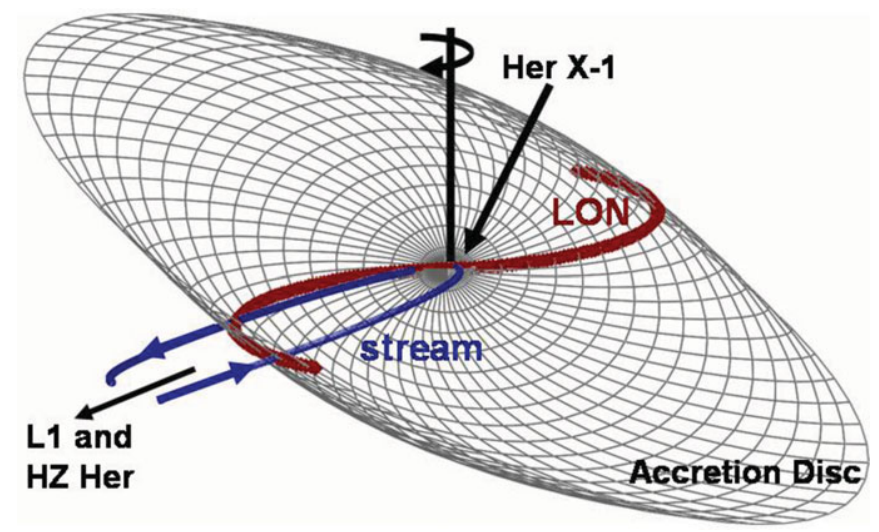

Figure 1. Binary geometry for Her X-1 in the binary reference frame, with wire-frame model for the accretion disk. The line-of-nodes of the disk is the heavy S-shaped line; the accretion stream trajectory is the line with arrows coming from HZ Her and passing near the neutron star. The direction of rotation of the disk in the binary frame is shown by the circular arrow around the z-axis above the neutron star. 
to a few hours, as described in the observational study of dips by Igna \& Leahy(2011). The upper envelope of the points in Figure 2 is the 35-day cycle, without dips. Previous studies of 35-day cycle using the RXTE/ASM data or other low sensitivity instruments could not separate out dips, and as a result, obtained a strongly biased 35-day light curve. Among the new features seen in the RXTE/PCA 35-day cycle light curve are: smooth rise and fall for main high state; a sharp rise and sharp fall for short high state.

\section{Accretion dips}

Accretion dips can be identified by softness ratio (SR) (2-4 keV count rate divided by $9-20 \mathrm{keV}$ count rate). Softness ratio is more useful than its inverse, hardness ratio, because of the strong low energy caused by the dips which gives very small $2-4 \mathrm{keV}$ count rates which can have large error bars. The changing SR can be compared with spectral models, showing cold matter absorption with column densities up to $10^{2} 4 \mathrm{~cm}^{-} 2$.

Light curves were constructed with all RXTE/PCA data for either main high or for short high plotted vs. orbital phase. These show that both main and short high have numerous dips, with more dips at later orbital phases. The average fraction of time with dips for $\mathrm{MH}$ is $28 \%$, with a minimum fraction at orbital phase 0.25 . The average fraction of time with dips for $\mathrm{SH}$ is much higher at $62 \%$, with a minimum at orbital phase 0.35 .

The dips were examined in detail by Igna \& Leahy(2011). Dips can be simple or complex We measured 370 new dips (about 10 times more than previous studies) Dip durations range from $1 \mathrm{~min}$ to several hours. Dip occurrence for long dips (>40 min) compared to short dips $(<40 \mathrm{~min})$ is the same vs. orbital period or 35-day period Observed dips occur at irregular intervals but are clustered regularly in the 35-day vs. orbital phase plot.

We construct a dips production model based on occulation by the accretion stream. See Igna \& Leahy(2012) for details. Roche lobe overflow produces a stream, such as modeled

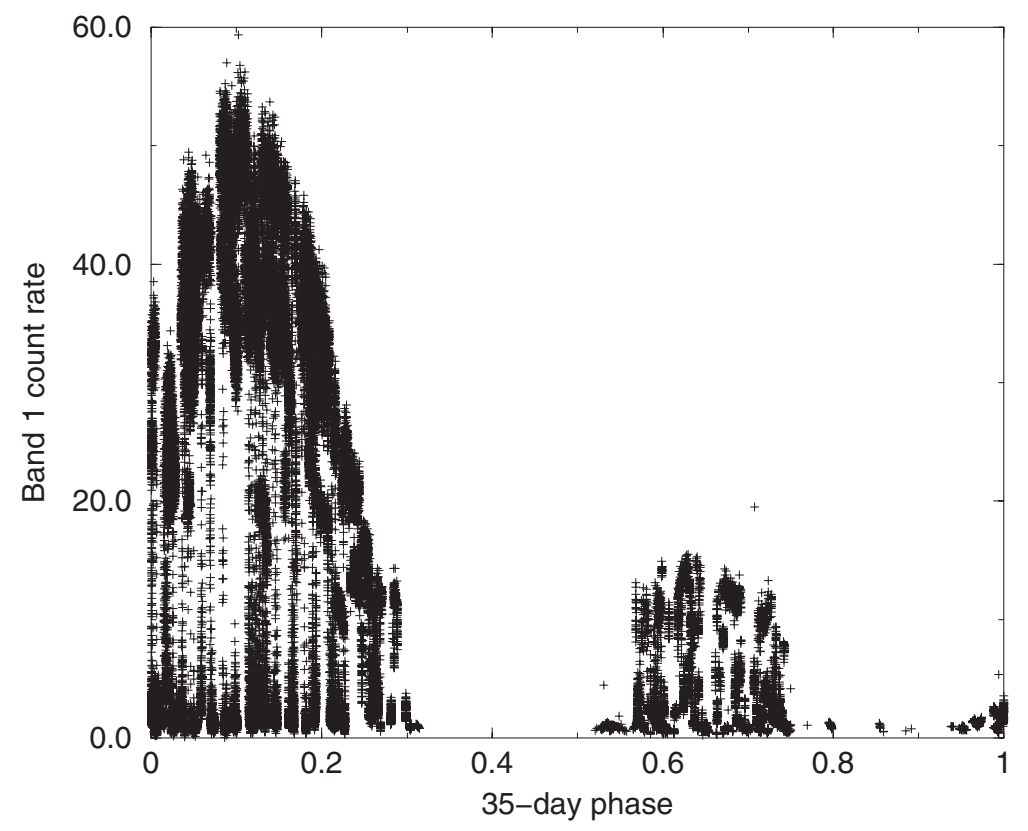

Figure 2. 35-day phased RXTE PCA lightcurve. 
by Lubow \& Shu(1975). We calculate the stream trajectory in the Roche potential and find where it intersects the tilted-twisted disk surface. This intersection point is the stream impact (splash region) and can block the observers line-of-sight (l.o.s.) to the neutron star, depending on disk and observer orientation Blockage of line-of-sight by splash region results in a dip. Occurrence times for model dips including disk rotation, binary orbit, and moving impact point on the disk were calculated. Whether a dip occurs depends on how large is the splash region. A size of $0.02 \mathrm{a}$ fits the observed number and timing of dips.

\section{Summary}

Over 1.5 million seconds of RXTE/PCA data on Her X-1 over about 10 years covering parts of 37 different 35-day cycles have been analysed for accretion dips. We find that dips are a strong function of orbital phase in main high and short high states, and that dips are much more frequent in short high state $(62 \%$ fraction of time for short high vs. $28 \%$ fraction in main high).

We have studied dip production by the accretion stream. In particular, the accretion stream impacts the accretion disk in the orbital plane, but the impact region is large enough to extend above the plane into the observer's line-of-sight (which is approximately 5 degrees above the plane). Our calculation of the accretion stream trajectory with a moving disk with a twisted and tilted shape gives an an impact region which moves around considerably (and with large jumps) as a function of time. The resulting position of the impact region and its location with respect to the observer's line-of-sight provided a good description of observed accretion dips.

\section{References}

Gerend, D. \& Boynton, P. E. 1976, The Astrophysical Journal, 209, 562

Igna, C. D. 2011, PhD. Thesis, University of Calgary

Igna, C. D. \& Leahy, D. A. 2011, Monthly Notices of the Royal Astronomical Society, 418, 2283

Igna, C. D. \& Leahy, D. A. 2012, Monthly Notices of the Royal Astronomical Society, 425, 8

Leahy, D. A. 2002, Monthly Notices of the Royal Astronomical Society, 334, 847

Leahy, D. A. 2003, Monthly Notices of the Royal Astronomical Society, 342, 446

Leahy, D. A. 2004, Monthly Notices of the Royal Astronomical Society, 348, 932

Leahy, D. A. 2004, The Astrophysical Journal, 613, 517

Leahy, D. A. \& Igna, C. D. 2010, The Astrophysical Journal, 713, 318

Leahy, D. A. \& Igna, C. 2011, The Astrophysical Journal, 736, 74

Lubow, S. H. \& Shu, F. H. 1975, The Astrophysical Journal, 198, 383

Petterson, J. A. 1975, The Astrophysical Journal Letters, 201, L61

Scott, D. M. \& Leahy, D. A. 1999, The Astrophysical Journal, 510, 974

Scott, D. M., Leahy, D. A., \& Wilson, R. B. 2000, The Astrophysical Journal, 539, 392 